\section{Forschende \\ Komplementärmedizin \\ Uissenschto Praxis Perspektiven}

\title{
Der Darm - Dirigator über leibliches Wohl und Übel
}

\author{
10. Dezember 2005, Friedrich-Schiller-Universität Jena
}

Gast-Herausgeber: C. Uhlemann, Jena

Zum 7. Male jährte sich im Dezember 2005 die Zusammenkunft von Naturheilkundlern und Schulmedizinern in Jena anlässlich der Symposiumsreihe "Zur Ratio und Plausibilität in der Naturheilkunde». Das Thema lautete: "Der Darm - Dirigator über leibliches Wohl und Übel». Dass der Darm nicht nur in der Naturheilkunde ein zentraler Aspekt diagnostischen und therapeutischen Denkens und Handelns ist, sondern auch in der modernen, schulmedizinisch geprägten Denkweise mehr und mehr in den Vordergrund rückt, wird in Publikationen und wissenschaftlichen Veranstaltungen der Gastroenterologen evident. Es ist daher sowohl vernünftig als auch dringend notwendig, wie auf der 60 Jahrestagung der Deutschen Gesellschaft für Verdauungs- und Stoffwechselerkrankungen gefordert, dass traditionelle Abteilungsstrukturen aufgelöst und eine interdisziplinäre Viszeralmedizin geschaffen wird, die je nach Patientenprofil Grundlagenwissenschaftler, Kliniker, Mikrobiologen, Rehabilitationsmediziner, Versorgungsmediziner und Immunologen vereint.

Mit einer Länge von etwa 6 m, einer Oberfläche von etwa $200 \mathrm{~m}^{2}$ und mehr Nervenzellen als das Gehirn beeinflusst das grösste Verdauungsorgan unmittelbar unsere Gesundheit. Zellen der Darmwand haben eine enorme immunologische Potenz, insofern ist der Darm wesentlich für eine gut funktionierende Abwehr. In der Naturheilkunde war und ist der Darm Ausgangs- sowie Endpunkt relevanten leibseelischen Wohls oder Übels. Ein häufig verwendeter Ausspruch der Naturheilkundler, "Der Tod sitzt im Darm", fokussiert diesen Sachverhalt.

Unter naturheilkundlichen Diagnostikprinzipien ist die Verdauung eine der Vogler'schen Grundfunktionen. Was für ein ganzheitliches, integratives System im Sinne eines biopsychosozialen Bedingungsgefüges der Darm bzw. die Bauchorgane sind, drückt sich auch in zahlreichen Redewendungen aus: man entscheidet "aus dem Bauch heraus", "verdaut" ein Problem, eine Sache bereitet "Bauchschmerzen", es gibt "Bauchdenker" und die berühmten "Schmetterlinge im Bauch» beim ersten Verliebtsein. Dann mobilisieren die Afferenzen dieses physiologischen Regelsystems alle emotional modulierten Efferenzen.

Das Symposium beleuchtete zunächst aus neurophysiologischer Sicht die Autarkie des Darmes (intramurales Nervengeflecht) und die Steuerung der Motorik und Sensorik, wobei die sog. Vagusafferenz für zahlreiche unspezifische Schmerzsyndrome eine Erklärung geben könnte. Danach wurde die These "der Darm - das 2. Gehirn» erläutert: Dazu wurde die neurobiologisch verankerte reziproke Kommunikation zwischen Gehirn und Darm (brain-gut axis) dargestellt und wie sympathische und parasympathische Wege in diese eingreifen.

Als Kliniker muss man sich die Frage stellen, warum das komplizierte Gefüge des Darms bei den meisten Menschen so gut funktioniert. Es ist die neuronale Regulation (Hirn als organisierendes Prinzip!) mit ihren psychischen und sozialen Einflussfaktoren, die über Kranksein oder Nichtkranksein entscheidet, also warum der eine krank wird und der andere nicht.

Komplettierend wurden aus sog. "somatischer» Sicht Aussagen zur Ätiologie und Pathogenese sowie zur Beeinflussbarkeit gestörter Darmfunktionen gemacht, dabei stand das Reizdarmsyndrom im Vorder- grund. Moderne somatische Therapiestrategien integrieren auch Massnahmen, die eine psychophysische Spannungsregulation herbeiführen (viszerale Analgetika, Prokinetika). Deren Wirkung ist jener von neuroendokrinalen Transmittern durchaus vergleichbar.

Die klassische Naturheilkunde hat langjährige gute Erfahrungen mit regulativen Interventionen. Pflanzen, die seit Jahrhunderten angewandt werden, um die Verdauung zu beeinflussen, und deren Wirksamkeit empirisch nachgewiesen ist, sind nicht nur praktizierenden Ärzten bekannt, sondern nehmen auch den Rang einer Volksmedizin ein: Karminativa (Kümmel, Fenchel, Anis) und Amara (Enzian, Wermut) werden in vielen Haushalten verwandt. Auch ärztlich geleitetes Fasten kann zu einer temporären Entlastung des Darms und seiner Schonung führen.

Eine weitere probate, wirksame und seit Jahrzehnten bewährte Massnahme zur Beeinflussung gestörter Darmfunktionen kann über sog. Reflexzonen erfolgen. Dabei werden die reflektorischen Verbindungen zwischen einzelnen Organsystemen, der Körperdecke und den inneren Organen sowie zwischen Peripherie - Viszerum und ZNS ausgenutzt. Ebenen der Einflussnahme sind Bindegewebszonen, Schröpfzonen, Meridiane, Somatotopien und die Schleimhaut des Darmes.

Auch neuraltherapeutische Interventionen zur Beeinflussung sympathischer bzw. parasympathischer Dysregulationen sind zweckmässig. In diesem Kontext ist es bedeutsam, dass die viszeralen Organe eine duale sensorische Innervation (spinale und vagale Afferenzen) erfahren, die den Darm, wenn er einmal gereizt ist, zu einem Orkan im Menschen werden lassen.

C. Uhlemann, Jena

\section{Zur Innervation des Darms}

Hans-Georg Schaible

Institut für Physiologie

Friedrich-Schiller-Universität Jena

Der Darm besitzt eine komplexe Innervation. Hierzu gehören (a) das Darmnervensystem (DNS), (b) sympathische und parasympathische Nervenfasern des autonomen Nervensystems (ANS), (c) afferente sensorische Nervenfasern. Nervenfasern haben vor allem eine modulierende Funktion, denn Grundfunktionen des Darms werden durch die glatte Muskulatur und durch Mukosazellen wahrgenommen. Die Peristaltik hat die Aufgabe, den Inhalt von Magen und Darm zu durchmischen und von oral nach aboral zu transportieren. Man unterscheidet mehrere Unterformen der Darmbewegungen. Die propulsive und nicht-propulsive Peristaltik besteht darin, dass sich ein Darmabschnitt kontrahiert und der benachbarte Darmabschnitt erschlafft. Dabei bewirkt die propulsive Peristaltik einen Weitertransport des Inhalts, die nicht-propulsive Peristaltik eine Durchmischung. Diese Formen der Peristaltik kommen in Ösophagus, Magen und Dünndarm vor. Ferner zeigen der Dünn- und Dickdarm rhythmische Segmentationen, die der Durchmischung des Darminhalts dienen.

\section{KARGER}

(C) 2006 S. Karger GmbH, Freiburg

Fax +497614520714

E-mail Information@Karger.de www.karger.com/fok 
Schliesslich bewirken Pendelbewegungen des Dünn- und Dickdarms eine umfangreiche Verschiebung des Darms über seinen Inhalt. Die Sphinkter sind tonisch kontrahiert. Diese Darmbewegungen werden von der glatten Muskulatu selbst generiert, in einem für den jeweiligen Magen-Darm-Abschnitt typischen Rhythmus. Dafür sind Schrittmacherzellen verantwortlich, in denen es in regelmässigen zeitlichen Abständen zu überschwelligen Depolarisationen kommt. Von diesen Schrittmacherzellen aus wird die Depolarisation durch Gap Junctions in angrenzende Zellen der glatten Muskulatur weitergeleitet. Bedingt durch diese Aktivität kontrahiert sich der Magen etwa 3-mal pro Minute, das Duodenum etwa 12-mal pro Minute, das Ileum etwa 8-mal pro Minute. Die spontane Depolarisation in Schrittmacherzellen kommt durch periodische Erhöhungen der Natrium- und Calciumleitfähigkeit zustande. Wird dabe die Schwelle erreicht, werden Aktionspotentiale ausgelöst, die durch einen Calciumeinstrom durch spannungsabhängige L-Typ-Calciumkanäle entstehen Das DNS greift in diese Vorgänge ein. Während die nicht-propulsive Peristal tik des Dünndarms durch den myogenen Rhythmus entsteht, wird die propulsive Dünndarmperistaltik durch das DNS gesteuert. Dieses sorgt dafür, dass der Darminhalt von oral nach aboral transportiert wird. Das ANS nimmt die übergeordnete Koordination der Peristaltik wahr, die in das Gesamtverhalten des Organismus integriert ist. Auch im Dickdarm besteht eine hierarchische Steuerung der Peristaltik. Nicht-propulsive Peristaltik entsteht durch Schrittmacherzellen, die sich im mittleren Dickdarmabschnitt befinden. Propulsive Massenbewegungen, die 3- bis 4-mal täglich zwischen den Mahlzeiten erzeugt werden, stehen unter dem Einfluss des ANS.

Das Darmnervensystem

Dieses besteht aus (a) afferenten Neuronen, die Signale aus dem Darm aufnehmen, (b) Motoneuronen, die die Peristaltik beeinflussen, und (c) Interneuronen, die zwischen die afferenten Neurone und die Motoneurone geschaltet sind. Die Neurone befinden sich im Plexus myentericus, der zwischen den Schichten der Ring- und Längsmuskulatur liegt, und im Plexus submucosus, der zwischen der Ringmuskulatur und der Submucosa liegt. Beide Plexus kom munizieren miteinander. Das DNS des Menschen besteht aus ca. $10^{8}$ Nervenzellen. Dieses Nervensystem ist so organisiert, dass es lokale Reflexbögen bildet. Afferente Neurone projizieren zu lokalen Interneuronen und Motoneuronen, ohne Verbindung zum ZNS aufzunehmen. Das DNS ist also ein komplettes Nervensystem, das im Darm lokale Vorgänge koordiniert. Es steuert den Ablauf der Motilität (z.B. den Transport von oral nach aboral), Sekretionsund Resorptionsvorgänge, endokrine Prozesse und die lokale Durchblutung.

\section{Das periphere autonome Nervensystem}

Präganglionäre Symapthikusfasern ziehen vom Thorakal- und Lumbalmark über die Vorderwurzeln zum Grenzstrang. Entweder sie passieren den Grenzstrang ohne Umschaltung und ziehen zu den sympathischen Ganglien, wo eine Umschaltung auf postganglionäre Neurone stattfindet, oder sie enden im Grenzstrang selbst an postganglionären Neuronen. Präganglionäre Parasympathikusfasern verlassen das ZNS über den N. vagus oder aus dem Sakralmark. Die Umschaltung auf postganglionäre parasympathische Neurone erfolgt in Ganglien, die in den Organen selbst liegen. Die meisten Organe sind sowoh sympathisch als auch parasympathisch innerviert. Die Gefässe jedoch sind mi Ausnahme der Gefässe in den Genitalorganen nur sympathisch innerviert. Die postganglionären Fasern nehmen Kontakt zum DNS auf. Im Magen-DarmTrakt haben die parasympathischen Fasern wichtige Funktionen. Parasympathische Fasern fördern die Peristaltik und die Tätigkeit von Drüsenepithelien und unterstützen die Wirkung endokriner Zellen. Beispielsweise stimulieren Vagusfasern die Gastrinfreisetzung und die Belegzellen des Magens, die $\mathrm{HCl}$ in das Magenlumen freisetzen. Dagegen hat der Sympathikus nur eine schwache (der Vaguswirkung entgegengesetzte) Wirkung auf die Darmmuskulatur und die Drüsenepithelien, aber er aktiviert die Sphinktermuskulatur. Wichtig is die sympathische Steuerung der Widerstands- und Kapazitätsgefässe im Magen-Darm-Bereich im Sinne der allgemeinen Blutdruckregulation.

Afferente sensorische Nervenfasern des Magen-Darm-Bereichs mit Projektion zum ZNS

Bei der sensorischen Innervation mit projizierenden Afferenzen liegt für die Viszeralorgane die Besonderheit vor, dass jedes Organ durch zwei Nerven innerviert wird: entweder durch spinale Nervenfasern und Vagusafferenzen oder durch spinale Nervenfasern und Afferenzen im Pelvicusnerven. Spinale Afferenzen ziehen durch den Grenzstrang und die paravertebralen Ganglien hindurch und treten über die Hinterwurzel in das Rückenmark ein. Ihre Zellkörper liegen wie die aller somatischer Afferenzen in den Hinterwurzelganglien. Die Axone können Kollateralen in die prävertebralen Ganglien abgeben. Vollständig geklärt ist die Funktion der spinalen und vagalen Afferenzen nicht. Die spinalen Afferenzen lösen spinale Reflexe und Schmerzempfindungen aus. $\mathrm{Ob}$ andere Empfindungen durch Reizung spinaler Afferenzen hervorgerufen werden, ist unklar. Vagale Afferenzen lösen ebenfalls Reflexe aus, aber unklar ist, ob sie Sinnesempfindungen bewirken. Schmerzempfindungen im Viszeralbereich sind durch mehrere Besonderheiten charakterisiert. Sie sind in der Regel diffus und schlecht lokalisiert, und sie werden häufig in somatische Strukturen übertragen. Viszerale Schmerzen haben eine ausgeprägte emotionale und vegetative Komponente. Sie werden ausgelöst durch die Überdehnung von Hohlorganen, Zug am Mesenterium, Entzündung und Ischämie. Dagegen rufen Schnittläsionen und Quetschungen viszeraler Gewebe keine Schmerzen aus. Die diffuse Natur der Schmerzen und die Übertragung in somatische Gewebe wird durch die Verarbeitung im Rückenmark erklärt. Hie findet man eine Konvergenz von viszeralen und somatischen Afferenzen auf dieselben Neurone. Das Gehirn lokalisiert aufsteigende Information in die Haut statt in die Viszeralorgane.

Die Reizung spinaler Afferenzen löst verschiedene Reflexe aus. Viszerosomatische Reflexe bringen die Muskulatur zur Kontraktion (z.B. der Hartspann bei entzündlichen Erkrankungen im Viszeralbereich), und viszerokutane Reflexe bewirken Änderungen der Hautdurchblutung und Schweisssekretion be Reizung viszeraler Organe. Die viszeralen Afferenzen können aufgrund ihre Antwortschwelle für mechanische Reize in niederschwellige, hochschwellige und mechanoinsensitive Afferenzen eingeteilt werden. Hochschwellige Rezeptoren werden als klassische Nozizeptoren angesehen, da sie nur bei potentiel schmerzhafter mechanischer Reizung (z.B. Dehnung eines Hohlorgans) ansprechen. Die mechanoinsensitiven Fasern werden als «schlafende Nozizeptoren» betrachtet. Diese Fasern reagieren auf mechanische Reizung des normalen Gewebes überhaupt nicht, aber sie werden mechanosensitiv, wenn sie im Laufe eines Entzündungsprozesses sensibilisiert werden. Die meisten Fasern sind allerdings niederschwellig. Typischerweise werden diese Fasern bereits be nicht schmerzhafter mechanischer Reizung viszeraler Organe aktiviert, doch antworten sie wesentlich stärker bei noxischen Reizintensitäten. Es ist nich endgültig geklärt, ob diese Fasern auch Nozizeptoren darstellen. Dafür spricht dass sie durch ihre Entladungseigenschaften die Stärke noxischer Reize kodieren können und dass sie, da sie polymodal sind, durch Entzündungsprozesse genauso wie hochschwellige Nozizeptoren sensibilisiert werden können. Diskutiert wird auch, dass solche Fasern unangenehme Empfindungen unspezifischer Art auslösen können.

Einem Teil der Vagusafferenzen wird eine besondere Rolle beim Körperschutz zugemessen. Insgesamt enthält der Vagus ca. 80\% afferente Fasern, während nur etwa $20 \%$ der Vagusfasern parasympathisch efferent sind. Viele Vagusafferenzen haben spezifische Aufgaben in der Vermittlung von respiratorischen, kardialen und gastrointestinalen Reflexen. Andererseits stehen Vagusafferenzen im Dienst des Körperschutzes. Besonders Vagusafferenzen aus dem Be reich der Leber und des oberen Gastrointestinaltrakts (GIT) induzieren eine sog. Krankheitsantwort, bestehend aus allgemeinen Krankheitssymptomen und einer generalisierten Hyperalgesie. Diese Vagusafferenzen werden durch entzündliche Vorgänge erregt, und sie aktivieren Neurone im Hirnstamm, die ihrerseits zum Hypothalamus projizieren. Nach einer subdiaphragmatischen Vagotomie kann eine Krankheitsantwort, die vom Intestinalbereich ausgelös werden kann, ausbleiben. Interessanterweise können die Vagusafferenzen auch weit reichende Auswirkungen auf pathologische Prozesse in somatischen Strukturen haben.

Zusammenfassend bleibt festzustellen, dass der GIT in seinen Grundfunktionen autonom ist. Was in unserem GIT funktionell geschieht, ist uns in der Regel nicht bewusst. Der GIT ist aber sensorisch und efferent mit dem ZNS verbunden, so dass die gastrointestinalen Grundfunktionen in den Verhaltenskontext eingebunden werden. Besondere Bedeutung haben hier auch die Warnsysteme, die der Abwehr von Körperschädigungen dienen. Dazu gehören sowohl Spinalnerven, die nozizeptive Reflexe und Schmerzempfindungen auslösen, als auch Vagusafferenzen, die eine Krankheitsreaktion induzieren.

\section{Literatur}

Bielefeldt K, Gebhart GF: Visceral pain: basic mechanisms; in McMahon SB, Koltzenburg M (eds): Elsevier Wall and Melzack's Textbook of Pain. Churchill Livingstone, Elsevier, 2005, pp 721-736.

Jänig W: Integrative Action of the Autonomic Nervous System: Neurobiology of Homeostasis. Cambridge, Cambridge University Press, 2006.

\section{Wie beeinflusst das Gehirn den Darm und der Darm das Gehirn?}

\section{Supported by the German Research Foundation}

Prof. Dr. Wilfrid Jänig

Physiologisches Institut

Christian-Albrechts-Universität zu Kiel

Die Hauptfunktionen des Gastrointestinaltraktes (GIT) sind Aufnahme Transport, enzymatischer Abbau und Resorption nutritiver Substanzen, Regulation der Flüssigkeitsbalance, Abwehr des Körpers gegen toxische Substanzen und Ausscheidung von Abfallprodukten. Diese Funktionen werden durch 
verschiedene Effektorzellen des GIT ausgeübt (glatte nichtvaskuläre Muskelzellen, sekretorische Epithelien, interstitielle Zellen von Cajal, endokrine Zellen, Zellen des Immunsystems [GALT, gut associated lymphoid tissue]). Das Darmnervensystem (DNS) ist ein spezialisiertes autonomes Nervensystem, welches aus Motoneuronen, intrinsischen primär afferenten Neuronen un Interneuronen besteht und die Aktivität dieser Effektorzellen reguliert. Dieses führt zu einer zeitlich und räumlich koordinierten Aktivität der Funktionen des GIT, die unabhängig vom Gehirn ablaufen kann [1].

\section{A. Reziproke Verbindungen zwischen GIT und Gehirn}

Die globalen Funktionen der Kontrolle des GIT durch das Gehirn sind biologisch die Anpassung des GIT an die verschiedenen Verhaltensweisen des Organismus und die Regulation der Energiebalance und damit des Körpergewichts (dieses schliesst Sättigung und Hunger ein) [3, 4].

I. Diese zentralnervöse Kontrolle ist besonders ausgeprägt am Eingang (oral) und am Ausgang (anal) des GIT. Sie besteht aus folgenden efferenten Komponenten: (1) Parasympathische Systeme, die ihren Ursprung im Nucleus dorsalis des N. vagus (NDNX) haben und in diesem Kerngebiet topographisch ange ordnet sind (Ösophagus, Magen, Dünndarm). Diese parasympathischen Systeme sind funktionell nach ihren Zielzellen in Untersysteme differenziert. Die Aktivierung individueller parasympathischer Systeme im NDNX führt zu Förderung oder Hemmung der Motilität (z.B. des Magens, Duodenums ode Ileum-Jejunums), zur Sekretion der Belegzellen des Magens, der Mukosa des Duodenums, Ileum-Jejunums, oder des exokrinen Pankreas, oder zur Freiset zung von Hormonen (z.B. Gastrin aus G-Zellen oder Sekretin aus S-Zellen der Mukosa des Duodenums; Insulin aus den Langerhans'schen Zellen des Pankreas) [5]. (2) Parasympathische Systeme im Sakralmark, die wichtig für die Regulation von Kontinenz und Entleerung des Enddarmes sind. (3) Sympathische nichtvasokonstriktorisch tätige Systeme, die ihren Ursprung im thorakolumbalen Rückenmark haben. Aktivierung dieser Systeme führt zur Hemmung der Motilität und der Sekretion im GIT. (4) Blutgefässe des GIT, besonders in der Mukosa, stehen zusätzlich zur lokalen Kontrolle durch das DNS direkt unter der Kontrolle des Gehirns über viszerale Vasokonstriktorneurone, die ebenfalls im Thorakolumbalmark repräsentiert sind. Diese zentralnervöse Kontrolle der Blutgefässe ist wichtig für die Regulation des arteriellen Blutdrucks [2].

II. Die afferente Information über die Prozesse im GIT zum Gehirn wird übe verschiedene neuronale, hormonelle und humorale Kanäle vermittelt $[2,6]$

(1) Viszerale afferente Neurone projizieren durch den N. vagus vom GIT zum Nucleus tractus solitarii (NTS). Sie werden erregt durch mechanische Reize (Dehnung, Kontraktion, Scherreize auf der Mukosa), chemische intraluminal Reize (z.B. Glukose, Lipide) und vermutlich andere Reize. Sie haben bis au die afferente Innervation des proximalen Ösophagus keine nozizeptive Funktion. Diese Afferenzen sind wichtig für die Regulation gastrointestinaler Funk tionen und die schnelle Sättigung. (2) Sakrale viszerale afferente Neurone. Sie sind wichtig für die Regulation von Entleerung und Kontinenz des Enddarmes sowie für die nichtschmerzhaften und schmerzhaften Empfindungen, die vom Enddarm ausgelöst werden können. (3) Thorakolumbale viszerale Afferenzen. Diese Afferenzen sind wichtig für die Erzeugung von Schmerzen und anderen unangenehmen Empfindungen des GIT und für die Erzeugung von protektiven Reflexen. (4) Hormone des GIT werden ins Blut ausgeschüttet und wirken über die Area postrema (AP) auf Neurone im NTS und NDNX (z.B. Cholezystokinin aus Mukosazellen, Glucagon-like peptide 1 [GL-1] aus Mukosazellen des Dünndarms) und auf Neurone im dem Nucleus arcuatus im Hypothalamus (z.B. Ghrelin aus der Mukosa des Magens, Insulin und Pancreatic Polypeptide [PP] aus dem Pankreas). Die hormonellen Rückmeldungen (zusammen mit der hormonellen Rückmeldung vom Fettgewebe durch das Peptid Leptin) sind wichtig für die Lang- und Kurzzeitregulation des Metabolismus (und damit der Energiespeicher [Kohlehydrate in der Leber, die schnell verfügbar sind; Fettgewebe, welches einer Langzeitregulation unterliegt]), der Nahrungsaufnahme und damit des Körpergewichts. Integrale Bestandteile die ser Regulation sind Sättigung und Hunger [3, 4]. (5) Die Neurone im Nucleus arcuatus und vermutlich im NTS und NDNX bekommen auch direkte nutritive Signale über die Glukose- und Lipidkonzentrationen im Blut.

\section{B. Der dorsale vagale Komplex}

I. Organisation des dorsalen vagalen Komplexes: Die Unterkerne des NTS, zu denen die gastrointestinalen (vagalen) viszeralen Afferenzen projizieren, der ventral lokalisierte DMNX und die AP bilden zusammen den dorsalen vagalen Komplex (DVK). Afferenzen vom GIT projizieren topographisch zu Unterkerngebieten des NTS. Die Sekundärneurone im NTS projizieren zu den präganglionären Neuronen im NDNX und bilden auf diese Weise vagale intestinointestinale Reflexkreise aus, die nach ihren Funktionen spezifiziert sind (s. A.I.1). Der Überträgerstoff der vagalen Afferenzen ist Glutamat. Die Überträgerstoffe der NTS-Neurone zu den Neuronen im NDNX sind entweder Glutamat (erregende Synapsen) oder Gamma-amino-Buttersäure (GABA hemmend) oder vermutlich auch Noradrenalin (erregend oder hemmend). Sekundärneurone im NTS projizieren auch zu anderen Kerngebieten im unteren Hirnstamm, im oberen Hirnstamm und im Vorderhirn (welches den Hypotha- lamus einschliesst). Neurone in den meisten dieser Kerngebiete projizieren zum NTS und zum NDNX zurück und beeinflussen synaptisch die intestino-intestinalen Reflexe im DVK. Darüber hinaus gibt es im NTS und im NDNX erregende und hemmende Interneurone, über die die synaptische Übertragung lokal moduliert wird. Die präganglionären Neurone im DMNX sind topographisch in rostrokaudalen Zellsäulen organisiert. Neurone, die zum Magen durch die Rami gastrici projizieren sind medial lokalisiert, Neurone zum Dünndarm durch die Rami coeliaci lateral und Neurone, die durch den Leberast projizieren, liegen zwischen der medialen und lateralen Zellsäule [2,5].

II. Integration endokriner Signale durch die Area postrema in der neuronalen Kontrolle des GIT: Die Aktivität der Reflexkreise im DVK wird auch moduliert durch hormonelle Signale im Blut über die Area postrema (AP). Solche Signale sind z.B. Hormone vom GIT (GL-1, PP oder Peptid YY) oder andere Hormone (corticotropin-releasing Hormon [CRH], thyreotropin-releasing Hormon [TRH]) oder Cytokine (z.B. Tumor Necrosis Factor $\alpha$ [TNF $\alpha])$. Die AP and benachbarte mediale Teile des DVK haben keine vaskuläre Blut-HirnSchranke, so dass die Hormonsignale (Peptide) freien Zugang haben zu den Reflexkreisen im DVK. Die Peptidhormone, die über die AP auf die Reflexkreise im DVK wirken, repräsentieren vermutlich organisierende Prinzipien für globale Funktionen des Systems Hirn-GIT: CCK: Förderung von Verdauung und Sättigung; CRH: Antwort des GIT während Stress; GLP-1: Förderung von Sättigung; PP: antizipatorische Vorbereitung für Nahrungsaufnahme TNF $\alpha$ : Abwehr durch den GIT; TRH: Koordination von Thermoregulation und Regulation von Metabolismus [2].

C. Kontrolle des dorsalen vagalen Komplexes durch den oberen Hirnstamm und das Vorderhirn (Hypothalamus und Telenzephalon)

Die funktionell definierten Reflexkreise im DVK zwischen den afferenten Neuronen vom GIT und den präganglionären zum GIT sind die basalen Bausteine für das Gehirn in der Kontrolle der Funktionen des GIT. Diese Situation ist konzeptionell ähnlich wie die Rolle der vegetativen spinalen Reflexkreise in der Regulation von Blutgefässen (Blutdruckregulation, Thermoregulation), Herz (Blutdruckregulation), Beckenorganen, GIT und anderen Organen über spinale vegetative prä- und postganglionäre Neurone [2]. Die Reflexkreise im DVK sind unter der Kontrolle von Neuronen in der Medulla oblongata und in supramedullären Hirnzentren (sog. «exekutive» Neurone) wie z.B. in der ventrolateralen und ventromedialen Medulla, in den Nuclei parabrachiales und Kölliker-Fuse, in der pontinen Area 5, im Nucleus paraventricularis hypothalami, im lateralen Hypothalamus, im zentralen Nucleus der Amygdala, im Inselkortex oder im Präfrontalkortex. Diese Kerngebiete erhalten auch detaillierte afferente Information vom GIT und von anderen viszeralen Organen (über den NTS und über die zirkumventrikulären Organe) und von den somatischen Geweben (Haut, tiefe somatische Gewebe). Die exekutiven Neurone und die basalen vegetativen Reflexkreise im DVK, die mit dem GIT verknüpft sind, repräsentieren den internen Zustand des Organismus, sofern der GIT betroffen ist. Ein wichtiger Teil dieses internen Zustandes ist die hormonelle Rückkopplung über die AP und über den Hypothalamus (Nucleus arcuatus), die aus mehreren Komponenten besteht. Sie ist integriert in die Regulation des Metabolismus (Ernährung, Nahrungsaufnahme, Körpergewicht), Thermoregulation, Regulation der Flüssigkeitsmatrix, Regulation der Fortpflanzung und Aufzucht, die Regulation der Protektion des Körpers (Immunsystem) und der Abwehr, die Regulation der zirkadianen Rhythmik. Diese Regulationen sind homöostatisch. Sie werden fortlaufend an den externen Zustand des Organismus, der im Telenzephalon repräsentiert ist, angepasst durch das Telenzephalon (limbisches System und Neokortex).

\section{Literatur}

1 Furness JB: The Enteric Nervous System. Oxford, Blackwell Science, 2005.

2 Jänig W: The Integrative Action of the Autonomic Nervous System: Neurobiology of Homeostasis. Cambridge, Cambridge University Press, 2006.

3 Schwartz MW: Central nervous system regulation of feed intake. Obesity 2006; 14:1S-8S.

4 Schwartz MW, Porte D: Diabetes, obesity, and the brain. Science 2005 307:375-379.

5 Travagli RA, Hermann GE, Browning KN, Rogers C: Brain stem circuits regulating gastric function. Annu Rev Physiol 2006;68:279-305.

6 Undem B, Weinreich D (eds): Advances in Vagal Afferent Neurobiology. Boca Raton, CRC, 2005 . 


\section{"Essen und Trinken hält Leib und Seele zusammen» - Interaktionen zwischen Darm und Seele}

\author{
PD Dr. rer. nat. F. Frauke Musial \\ Innere Medizin V, Naturheilkunde u. Integrative Medizin \\ Universität Duisburg-Essen
}

Dass psychische Befindlichkeiten und emotionale Zustände, also im weitesten Sinne «seelische Faktoren» den Verdauungstrakt und damit unser Wohlbefinden nachhaltig beeinflussen können, erschliesst sich intuitiv bereits aus dem Volksmund: So beschreiben Ausdrücke wie «Liebe geht durch den Magen» oder «sich vor Angst in die Hose machen» die enge Verbindung zwischen psychischen Phänomenen und den Funktionen des Verdauungstraktes. Zusammenhänge zwischen psychischen Vorgängen und gastrointestinalen $\mathrm{Be}$ schwerden werden aber auch im Zusammenhang mit chronisch funktionellen Darmerkrankungen, also Erkrankungen des Verdauungstraktes, bei denen oftmals sehr beeinträchtigende Beschwerden und insbesondere Schmerzen ohne pathophysiologisches Korrelat auftreten, diskutiert. Einer der Pioniere au dem Gebiet der Erforschung des Zusammenhangs zwischen negativen Emotionen und Stress bei Reizdarmpatienten und gesunden Probanden war Thomas P. Almy in der Mitte des letzten Jahrhunderts [Überblick: Musial, 2002], der die Auswirkungen von Emotionen und verschiedenen Laborstressoren au die gastrointestinalen Funktionen untersuchte. In Almys Untersuchungen zeigte sich, dass die Kolon-Kontraktionsmuster bei den untersuchten Patienten häufig von denen gesunder Probanden abwichen. Dabei kamen sowohl ausgeprägtere, als auch abgeschwächte Kontraktionsmuster vor. Daraus wurde der Schluss gezogen, dass Reizdarmpatienten eine Störung der Kolonmotilität auf weisen, die in der Folge gastrointestinale Beschwerden wie Diarrhoen, Obstipation und Schmerzen verursacht. Dieser, als Motilitätshypothese bezeichnete Ansatz, führte dazu, dass die Aufzeichnung der gastrointestinalen Motilität Jahrzehnte lang die Methode der Wahl bei psychophysiologischen Untersuchungen im Verdauungstrakt war. Nach vielen Jahren der Erforschung dieses Ansatzes zeigt sich jedoch, dass die Ergebnisse prinzipiell sehr heterogen sind. Das primäre Postulat der Motilitätshypothese, dass es einen Zusammenhang zwischen Veränderungen der Motilitätsmuster und negativen Emotionen (z.B Trauer), akutem Stress (z.B. eine Prüfungssituation) und dauerhafter Belastung (z.B. Zeitdruck) gibt, wird heutzutage nicht prinzipiell bezweifelt, insgesamt jedoch bleibt der Zusammenhang zwischen gastrointestinaler Motilität und den spezifischen Symptomen, insbesondere Schmerzen, unklar.

Im Rahmen der anorektalen Funktionsdiagnostik werden in aller Regel die Wahrnehmungsschwellen für Stuhldrang und Schmerz mittels intraluminale Ballondehnung bestimmt. Einer der konsistentesten und ältesten Befunde ist, dass Patienten mit Reizdarmsyndrom (RDS) sehr viel früher die Schwelle für das «maximal tolerierte Volumen», ein Mass für die Schmerzschwelle, erreichen. Eine ähnliche Verschiebung dieser Schwelle findet sich im Magen bei Patienten mit nicht ulzeröser Dyspepsie und im Ösophagus bei Patienten mit nicht kardialem Thoraxschmerz. Dieser konsistente Befund wurde als Hinweis für eine «viszerale Hypersensibilität» von Patienten mit funktionellen Darmerkrankungen gewertet. Diese sog. Hypersensibilitätshypothese postuliert, dass Patienten mit funktionellen Darmerkrankungen eine gestörte Verarbeitung af ferenter Impulse bei der Perzeption intestinaler Reize aufweisen, so dass normale Motilitätsereignisse, aber auch andere Stimuli wie z.B. Säuresekretion, verstärkt wahrgenommen werden. Aus tierexperimentellen Befunden kann zumindest für einen Teil der Patienten eine Veränderung der Reizverarbeitung bereits auf der Ebene der zum ZNS leitenden Nervenfasern und damit bereit auf Rückenmarksebene angenommen werden [Übersicht: Jänig und Häbler, 2002]. Darüber hinaus sprechen Befunde zur zentralnervösen Schmerzverarbeitung dafür, dass zumindest bei einem Teil der Patienten die Wahrnehmung viszeraler Sensationen auch kortikal verändert ist. Ähnliche Befunde zeigen sich bei depressiven Patienten und lassen sich unter bestimmten Bedingungen auch bei gesunden Probanden unter Angst, Stress und Belastung induzieren.

Neurowissenschaftliche Erkenntnisse der letzten Jahre haben unser Wissen über die Funktion und Arbeitsweise sog. «Schmerznetzwerke» im Gehirn enorm erweitert. Die Grundlage für eine Schmerzempfindung ist zunächst eine Gewebsschädigung in der Körperperipherie. Diese Information wird über spezialisierte Nervenfasern zum Gehirn weitergeleitet. Das Gehirn hat nun die Aufgabe, diese wichtige Information über eine Bedrohung der körperlichen Integrität zu bewerten und - falls erforderlich - Verhaltensmassnahmen einzuleiten. Dieser Vorgang der Weiterverarbeitung im Gehirn führt erst zu der eigentlichen Schmerzwahrnehmung, die aus sensorisch-diskriminativen («es tut wenig/deutlich/sehr weh; der Schmerz ist schneidend/stechend/brennend»), emotionalen («es ist auszuhalten/unangenehm/schrecklich/unerträglich») und kognitiven («es ist nur vorübergehend / ich kann mich nicht konzentrieren / ich kann nicht arbeiten») Komponenten besteht. Die Information, die das Gehirn über Gewebsschädigungen in der Körperperipherie erhält, wird über zwei Wege weiterverarbeitet: Der erste Weg enkodiert Informationen über Art und
Ausmass der Gewebsschädigung und wird als das sensorisch-diskriminative Schmerzverarbeitungssystem bezeichnet. Es führt im Gehirn über den Thalamus zu denjenigen Strukturen auf der Grosshirnrinde, die auch Informationen über andere Empfindungen körperlicher Art, wie z.B. Berührung, Tastsinn usw. weiterverarbeiten (Areale SI, SII). Der zweite Weg führt vom Thalamus zu Gehirnstrukturen, die die gefühlsassoziierten Schmerzbewertungen vermitteln (anteriorer Gyrus Cinguli, Insula). Im Regelfall wird dieses zweite System intensitätsabhängig vom ersten System getriggert: Vermitteln die einströmenden Informationen eine eher leichte Gewebsschädigung, wird die Reaktion des zweiten Systems nur mässig ausfallen. Handelt es sich aber um einen intensiven Stimulus, wird auch das affektive Schmerzsystem stärker reagieren und zu einer Schmerzbewertung gelangen, die vermittelt, dass die Schmerzen schlimm, unerträglich und belastend sind und unbedingt beseitigt werden müssen.

Diese Zweiteilung der Schmerz verarbeitenden Systeme ist vor dem Hintergrund negativer Emotionen vor allem deshalb interessant, weil das emotionale Schmerzsystem aufmerksamkeitsabhängig arbeitet. Das bedeutet einerseits, dass Schmerzen unter Aufmerksamkeitsablenkung weniger intensiv wahrgenommen werden (z.B. Filme während einer Zahnbehandlung anschauen) heisst aber andererseits auch, dass dann, wenn unter Belastung Schmerzen als besonders behindernd oder einschränkend empfunden werden («Nein, nicht jetzt schon wieder Bauchschmerzen, ich muss doch gleich die Präsentation vorstellen ...»), diese Schmerzen noch unerträglicher werden. Viszerale Schmerzen zeichnen sich darüber hinaus durch eine Reihe von Besonderheiten aus: Sie sind dumpf, diffus und schwer lokalisierbar und oftmals von autonomen Reflexen begleitet. Sie treten häufig ohne bekannte strukturelle Schädigungen auf, und es kommen ausgedehnte, viszerosomatische Schmerzprojektionen vor. Autonome Funktionen und viszerale Informationen aus der Peripherie werden sowohl auf Rückenmarksebene wie auch im Thalamus mehrfach umgeschaltet und vorverarbeitet, bis die Information schliesslich im wichtigsten kortikalen autonomen Zentrum, der Insula, integriert wird [Jänig und Häbler, 2002].

Tatsächlich ist es so, dass viele Befunde bei Patienten mit funktionellen Darmerkrankungen nahe legen, dass die Aktivität dieses motivational-emotionalen Schmerzsystems deutlich erhöht ist und dass die Reizbewertung, zumindest für viszerale Schmerzen, vom eigentlichen Reizinput entkoppelt ist. Darüber hinaus liegen ähnliche Befunde für gesunde Probanden vor, bei denen gezeigt werden konnte, dass die Darbietung von emotional belastendem Bildmaterial die Reaktion auf experimentelle, viszerale Schmerzreize deutlich verstärkte. Noch interessanter wird die Beurteilung dieser Befunde vor dem Hintergrund, dass diejenigen Netzwerke im Gehirn, die Angst und Furchtreaktionen vermitteln, wie z.B. die Amygdala (der sog. «Mandelkern») mit ihren Projektionen, sich stark mit Hirnstrukturen überschneiden, die auch bei der emotionalen Verarbeitung von Schmerzen eine Rolle spielen [Dolan, 2002] Wahrscheinlich ist es biologisch durchaus sinnvoll, Situationen zu erkennen und $\mathrm{zu}$ antizipieren und entsprechend zu vermeiden, die potenziell gefährlich sind und damit auch eine Gefährdung der körperlichen Integrität nach sich ziehen können. Darüber hinaus scheinen Reaktionen wie z.B. die spontane Darmentleerung Bestandteil der sog. «Kampf-Flucht Reaktion» und damit integraler Bestandteil der Stressreaktion zu sein.

\section{Literatur}

Jänig W, Häbler H-J: Physiologie und Pathophysiologie viszeraler Schmerzen Schmerz 2002;16:429-445.

Musial F: Psychophysiologie des Reizdarmsyndroms. Schmerz 2002;16:452-459. Dolan RJ: Emotion, cognition, and behavior. Review. Science 2002;98(5596):1191-4.

\section{Bedeutung und Bedeutsamkeit eines gestörten Darms aus somatischer Sicht}

Priv.-Doz. Dr. med. habil. Uwe Will

Dep. für Gastroenterologie, Hepatologie und Allg. Innere Medizin SRH Wald-Klinikum Gera

\section{Differentialdiagnostik organischer Erkrankungen mit Symptomen des Reiz-} darmsyndroms

Klinische Alarmsymptome, die gegen das Vorliegen eines Reizdarmsyndroms (RDS) sprechen, sind unklarer Gewichtsverlust, Blutauflagerungen oder Blutbeimengungen im Stuhl und die Kombination der Schmerzsymptomatik mit Durchfall und Fieber als Hinweis für eine entzündliche Affektion des Gastrointestinums. Das fehlende Sistieren der gastrointestinalen Beschwerdesymptomatik in der nächtlichen Ruhephase sowie eine positive Familienanamnese mit Nachweis von Tumoren in der Eltern- oder Grosselterngeneration sollten eine sofortige gastroenterologische Diagnostik veranlassen. Eine umfassende Anamnese der Patienten mit der beschriebenen klinischen Symptomatik ist es- 
sentiell. Neben einer Familienanamnese von chronisch-entzündlichen Darmerkrankungen oder Tumorerkrankungen bzw. Pankreasaffektionen kann de Verdacht auf entzündliche Störungen des Gastrointestinaltraktes (GIT) durch gezielte Fragen nach Kontaminationen, Inokulationen oder der Reiseanamne se eruiert werden. Bei Nachweis einer unklaren Durchfallerkrankung sind in jedem Falle Stuhlproben zum Ausschluss einer infektiösen Gastroenteritis er forderlich. Hier erfolgt der Nachweis auf Typhus-, Paratyphus-, EnteritisRuhr- und pathogene E.-coli-Keime. Bei fehlendem Nachweis dieser pathogenen Krankheitserreger und einer chronischen Diarrhoe sollte an das Vorliegen einer Yersinose, einer Infektion mit Campylobacter jejunii, Lamblien ode Amöben gedacht werden. In diesen Fällen müssen die serologische und die entsprechende Stuhldiagnostik erfolgen. Chronisch-entzündliche Darmerkrankungen gehen mit Durchfällen einher, die wechselhaft mit Schleim oder Blut vermengt sein können. In diesen Fällen ist bei Erstdiagnostik eine Koloileoskopie mit Stufenbiopsien indiziert. Gewichtsverlust und okkulter Blutnachweis im Stuhl sind die Alarmsymptome für das Vorliegen eines Karzinoms im GIT. Bei Vorliegen dieser Symptomatik sollte zwingend eine Koloskopie zum Ausschluss des Kolonkarzinoms erfolgen. Als Minimaldiagnostik bei unklaren abdominellen Beschwerden wird laborchemisch die Bestimmung des CRP, des Blutbildes und der Blutsenkung als Parameter der infektiösen Enteritis neben einer sonographischen Diagnostik - die in jedem Fall durchzuführen ist - gefordert. Die sonographische Diagnostik als der verlängerte Arm des Untersuchers kann tumoröse Affektionen der parenchymatösen Organe sowie chronisch-entzündliche Affektionen am Pankreas detektieren. Neben diesen Erkrankungen ergibt sich sonographisch der Hinweis auf eine infektiöse Enteriti durch den Nachweis erweiterter flüssigkeitsgefüllter Dünndarmschlingen, gleichfalls können obstruktive Veränderungen am GIT durch den Nachweis einer pathologischen Kokarde (echoarme Wandverdickungen des Intestinums) gesehen werden. Ein pathologischer sonographischer Befund am Gastrointes tinum sollte in jedem Fall eine endoskopische Diagnostik nach sich ziehen. Die endoskopische Diagnostik beinhaltet eine Gastroduodenoskopie mit Dünndarmbiopsie zum Ausschluss einer glutensensitiven Enteropathie, die wie ein RDS imponieren kann [1]. Eine Koloileoskopie mit Stufenbiopsien ist zum Ausschluss einer mikroskopischen oder kollagenen Kolitis bzw. einer eosinophilen Kolitis als Hinweis auf ein nahrungsmittelallergisches Geschehen erforderlich. Erkrankungen, die mit einer gastrointestinalen Beschwerdesymptomatik ähnlich dem Reizdarm imponieren, sind zudem das Ligamentum-Arcuatum-Syndrom oder die chronisch-mesenteriale Ischämie. Beide Erkrankungen können durch eine Kombination der Sonographie mit der Duplexsonographie ausgeschlossen werden. Eine wichtige Stoffwechselstörung, die zu unklaren gastrointestinalen Beschwerden führt, ist die akute intermittierende Porphyrie. Zum Ausschluss der Porphyrie muss der Urin auf Porphyrine gesammelt werden. Nahrungsmittelallergien sind weiterführend durch den Prick-Test und spezielle serologische Untersuchungen zu verifizieren. Wenn diese diagnostische Kaskade ihren Abschluss gefunden hat und keine organischen Erkrankungen als Ursache für die Beschwerden zu eruieren waren, kann von einer funktionellen Störung des GIT ausgegangen werden.

\section{Das Reizdarmsyndrom (RDS)}

Epidemiologie: Die Prävalenz des RDS wird mit 14-18\% angegeben, Frauen erkranken etwa 3-mal häufiger als Männer. In der allgemeinärztlichen Praxi wird mit etwa 3\% der Patienten, die wegen eines RDS zum Hausarzt kommen, gerechnet. In einer gastroenterologischen Praxis macht dieses Patientenklientel etwa $35 \%$ aus. Nach Schätzungen wird angenommen, dass nur etwa $20 \%$ der symptomatischen Patienten einen Arzt aufsuchen. Die Definition des RDS wurde in den Rom-II-Kriterien definiert [2].

Bei Ausschluss einer organischen Erkrankung und dem Vorliegen von zwe der drei aufgeführten Symptome kann mit einer Wahrscheinlichkeit $>90 \%$ vom Vorliegen eines RDS ausgegangen werden. Die Differentialdiagnostik de RDS unterscheidet je nach der dominierenden klinischen Symptomatik zwischen dem Diarrhoe-Typ, dem Obstipations-Typ, dem alternierenden Typ und dem postinfektiösen Typ. Neben den gastrointestinalen Beschwerden können bei Patienten mit RDS häufig auch andere funktionelle Erkrankungen oder vegetative Dysfunktionen nachgewiesen werden. Tabelle 1 führt einige dieser assoziativen Beschwerdekomplexe auf.

\section{Tab. 1. Reizdarmsyndrom - Assoziation mit anderen Erkrankungen}

\section{- Angstsyndrom - Depression: bis 50\%}

- Sodbrennen, Aufstossen, Übelkeit, Globusgefüh

- Funktionelle Dyspepsie

- Kardiale Beschwerden

- Miktionsbeschwerden

- Fibromyalgie

- Chronisches Müdigkeitssyndrom - CFF

- Viszerale Hypersensitivität: $75 \%$

- Proctalgia fugax
Die Ätiologie des RDS ist multikausal. Neben einer gestörten Motilität wird eine viszerale Hypersensitivität als die Hauptursache für das RDS angenommen. Die Motilität des Gastrointestinums wird durch das enterale Nervensystem gesteuert, welches in einem komplexen Reflexbogen zwischen Darm, enteralem NS und ZNS die Modulation der Motorik am Gastrointestinum steuert. Beim RDS sind keine spezifischen Motilitätsmuster erkennbar, so dass komplexere Störungen des Reflexbogens bzw. Störungen zwischen dem enteralen und zentralen NS als Ursache für die Beschwerden diskutiert werden [3] Die Hypersensibilitätshypothese stützt sich auf eine Intoleranz bzw. verminderte Schmerzschwelle der Patienten mit Reizdarm für intraluminale Deh nungsreize. Als Ursache bei Hypersensibilität werden abgeklungene Entzündungen oder Medikamente bzw. Nahrungsmittel angenommen, die zu einer temporären Hyperalgesie führen, welche wiederum zu einer Aktivierung von Nozizeptoren auf spinaler Ebene führen, so dass es zu einer veränderten Perzeption intestinaler Afferenzen kommt [4]. Neben den Motilitäts- und Hypersensibilitätsmechanismen werden beim RDS noch immunologische und genetische kausalpathogenetische Besonderheiten nachgewiesen. Neben einem Cytokin-Gen Polymorphismus mit hohen Werten an proinflammatorischem TNF $\alpha$ und Interleukin(IL)-12 wurden bei Patienten geringere Werte von antiinflammatorischem IL-10 gefunden [4]. Auf die Beziehung des RDS zu Nahrungsmittelüberempfindlichkeiten weisen Untersuchungen von Zar hin, der zeigen konnte, dass der Antikörper Immunglobulin G 4 gegen Nahrungsmittel (Weizen, Schwein-, Lamm-, Rindfleisch) im Vergleich zur Kontrolle deutlich erhöht war $(\mathrm{p}<0,001)$. Eine über 6 Monate spezifische Nahrungskarenz für diese Nahrungsmittel liess Schmerzperzeption, die Compliance und die rektale Sensitivität bei diesen Patienten deutlich verbessern [7]. Auf eine Korrelation des immunologischen Phänomens eines vermehrten Nachweises von Mastzellen, Histamin und Prostaglandin E2 bei Patienten mit RDS weisen Barbara et al. [5] hin. Ein wichtiger pathogenetischer Faktor scheinen psychosoziale Faktoren zu sein. Es wird immer wieder auf die Assoziation mit spezifischen Persönlichkeitsvariablen bei ängstlichen Patienten mit RDS hingewiesen. So zeig sich, dass diese Patienten häufig mit depressiven Verstimmungen sowie Feindseligkeit reagieren, zudem kann eine gestörte Verarbeitung der subjektiven Reize (gelerntes Krankheitsverhalten) gesehen werden. Ein mangelndes Vertrauen in die Therapie und ein häufiger Arztwechsel führen nicht selten zur Chronifizierung der Beschwerden mit Entwicklung von psychoorganischen Syndromen, die oft therapierefraktäre Verläufe nehmen.

\section{Therapie des Reizdarmsyndroms}

Oberste Prämisse in der Therapie von Patienten mit Verdacht auf RDS ist die eingehende ärztliche Zuwendung und eine umfassende Ernährungsberatung. Da es sich bei den Patienten meist um ängstlich hyperreagible Patienten handelt, kann man durch Ausschluss einer organischen Erkrankung im Vorfeld unterschwellige Ängste bei den Patienten durch ein aufklärendes Gespräch minimieren. Wenn es gelingt, eine stabile, vertrauensvolle Arzt-Patient-Beziehung aufzubauen, ist eine Linderung der Beschwerden in bis zu 70\% der Fälle allein durch ein ärztliches Gespräch und eine Ernährungsberatung zu erzielen. Bei Hinweisen auf eine psychische Fehlentwicklung mit somatischer Komponente im Sinne eines RDS sollte in jedem Fall ein psychotherapeutischer Kollege in die Behandlung einbezogen werden. Die spezifischen Therapien des RDS richten sich nach dem entsprechenden Typ. Der Obstipations-Typ sollte mit ballaststoffreicher Kost mit Laxantien oder mit 5-Hydroxytryptamin-4 Agonisten (5HT-4) (Tegaserod) behandelt werden. Der Diarrhoe-Typ erfordert die Behandlung mit Probiotika, Loperamid oder Opiatanaloga. Bei Patienten, die vordergründig über Tenesmen klagen, kann ein Behandlungsversuch mit Karminativa oder Verdauungsenzymen versucht werden. Bei abdominellem Schmerz als vordergründiges Symptom kann mit Spasmolytika oder Antidepressiva (SSRI) bzw. mit dem 5-Hydroxytryptamin-3-Agonisten (5HT3) (Alansetron) ein Behandlungsversuch gemacht werden [3]. Neuere Therapieansätze mit Probiotika basieren auf der pathophysiologischen Erkenntnis, dass es bei Patienten mit RDS im Dünndarm zu einem bakteriellen Overgrowth bzw. einer bakteriellen Dyssynergie kommt, welche durch Probiotikagaben reguliert werden kann [6]. Seit 1989 sind 8 placebokontrollierte Studien mit der Gabe von Probiotika bei RDS publiziert. O’Mahony et al. konnten zeigen, dass durch Gabe von Bifidobacterium infantis und Lactobacillus die Schmerzperzeption und die Häufigkeit von Blähungen signifikant zu bessern waren [6].

\section{Literatur}

1 Wahnschaffe U, Ullrich R, Riecken EO, Schulzke JD: Celiac disease-like abnormalities in a subgroup of patients with irritable bowel syndrome. Gastroenterology 2001;121:1329-38.

2 Chey WD, Olden K, Carter E, Boyle J, Drossman D, Chang L: Utility of the Rome I and Rome II criteria for irritable bowel syndrome in U.S. women. Am J Gastroenterol 2002;97:2803-11.

3 Lea R, Hopkins V, Hastleton J, Houghton LA, Whorwell PJ: Diagnostic criteria for irritable bowel syndrome: utility and applicability in clinical practice. Digestion 2004;70:210-3 
4 van der Veek PP, van den Berg M, de Kroon YE, Verspaget HW, Masclee AA Role of tumor necrosis factor-alpha and interleukin-10 gene polymorphisms in irritable bowel syndrome. Am J Gastroenterol. 2005;100(11):2510-6.

5 Barbara G, Stanghellini V, De Giorgio R, Cremon C, Cottrell GS, Santini D, Pasquinelli G, Morselli-Labate AM, Grady EF, Bunnett NW, Collins SM, Corinaldesi R: Activated mast cells in proximity to colonic nerves correlate with abdominal pain in irritable bowel syndrome. Gastroenterology 2004;126(3):693-702.

6 O'Mahony et al: Lactobacilli and bifidobacterium in irritable bowel syndrome: symptom responses and relationship to cytokine profiles. Gastroenterology 2005 ; 128:541-551.

\section{Relevanz des Darmes in der klassischen Naturheilkunde und deren regulative Optionen}

Dr. Dr. Bernhard Uehleke

Abt. Naturheilkunde der Charite Universitätsmedizin

Campus Benjamin Franklin, Berlin

Die Darmfunktion wird in der Naturheilkunde in Zusammenhang mit anderen Organ- und Körperfunktion gesehen, und dementsprechend wird eine erweiterte Diagnostik und Anamnese erhoben. Dabei geht es zunächst um den Bewegungsapparat und die Haltung, wie sie besonders in den verschiedenen Bauchformen nach F.X. Mayr unterschieden werden. Rückenbeschwerden können in Zusammenhang mit gestörter Verdauung stehen. Therapeutisch werden durch Bewegung und Laufen reflektorisch prokinetische Wirkungen auf das Magen-Darm-System induziert. Es geht aber auch um die Ernährung in qualitativer und quantitativer Hinsicht und damit in Zusammenhang stehend auch Stoffwechsel und wiederum Konstitution. Gerade bei übergewichtigen Patienten werden aber auch benachbarte Organsysteme negativ beeinflusst, insbesondere die Lunge, das Herz und das urogenitale System und das Abflusssystem der unteren Extremität.

Über kutaneoviszerale Reflexzonen können Störungen des Magen-Darm-Systems erkennbar werden und dann durch Manipulationen wie Schröpfen oder Bindesgewebszonenmassage nach Dicke beeinflusst werden.

Im ganzheitlichen Sinn sind die vielschichtigen Wechselwirkungen zwischen Psyche und Darm zu berücksichtigen. In der Sichtweise traditioneller Medizinsysteme und insbesondere in der Traditionellen Europäischen Medizin stellt sich der Zusammenhang zwischen Darm und Psyche deutlicher dar als in der organbezogenen wissenschaftlichen Medizin. Eine ungestörte Darmfunktion ist für das psychische Wohlbefinden unerlässlich und selbst unbedeutend erscheinende Störungen wie Blähungen oder Obstipation stören. Umgekehrt stört übermässiger psychischer Stress jegliche normale Darmfunktion.

Dem Darmsystem direkt verbunden ist der grösste Teil unseres Immunsystems. Bei vielen Störungen des Immunsystems ist die Ursache im MagenDarm-System zu finden, dazu gehört auch eine unphysiologische Darmflora oder ein sog. «leaky gut», welches wiederum Folge einer chronischen Reizung sein kann.

Mit den klassischen Naturheilverfahren wie Hydro-Balneotherapie, Bewegungstherapie und Massagen, Ordnungstherapie und Ernährungstherapie un Heilfasten kann dementsprechend die gestörte Funktion günstig beeinflusst werden. Eine spezifischere Wirkung ist durch Phytotherapie und andere Hausmittel wie z.B. Heilerde zu erwarten. Viele Heilpflanzen stellen als Gewürze den Übergang zwischen Ernährung und Phytotherapie dar. Sie können in folgende Gruppen eingeteilt werden: Bitterstoffdrogen (Bitterstoffe, Gewürze Ätherisch-Öl-Drogen): dyspeptische Beschwerden; Leber-Galle-Mittel; Gerb stoffdrogen; entzündungshemmende Drogen; Karminativa/Spasmolytika; Laxantien; Quellmittel.

Bei funktionellen Verdauungsbeschwerden liegen meist komplexe Muster von Symptomen und Fehlfunktionen vor, die durchaus für eine Multi-Target-The rapie in Form von bewährten Heilpflanzenkombinationen sprechen. In Vergleichstudien konnte z.B. das Kombinationsarzneimittel Iberogast ${ }^{\circledR}$ (Steigerwald; Darmstadt, Deutschland) durchaus mit modernen pharmakologischen Ansätzen bezüglich der Wirksamkeit mithalten und zeigte sich bei der Verträglichkeit sogar überlegen [1]

Der in der Luvos ${ }^{\circledR}-$ Heilerde (Luvos-Just, Friedrichsdorf, Deutschland) enthaltene Löss ist besonders fein und besitzt ein sehr hohes Säurebindungsvermögen im Bereich chemisch definierter Antazida sowie ein sehr hohes Adsorptionsvermögen. In einer eigenen Beobachtungsstudie konnten wir feststellen, das viele Patienten unzufrieden mit den bisherigen Therapien sind und sich gerne einer Behandlung mit einem Naturheilmittel unterziehen. Es zeigte sich eine deutliche aber protrahiert einsetzende Linderung aller Symptome über eine Behandlungszeit von 6 Wochen. Damit scheint Heilerde über die antazide Wir kung hinaus Umstimmungen der Darmfunktion zu bewirken - möglicherweise auch über eine Beeinflussung der Darmflora - wie sie sonst in der Naturheilkunde durch Symbioselenkung und Ernährungsumstellung vorgenommen wird. Zusammenfassend bietet die Naturheilkunde vielfältige Therapieoptionen, die auch bei hartnäckigen Beschwerden und Störungen eine nachhaltige Besserung erwarten lassen.

\section{Literatur}

1 Rösch W, Vinson B, Sassin I: A randomised clinical trial comparing the efficacy of a herbal preparation STW 5 with the prokinetic drug cisapride in patients with dysmotility type of functional dyspepsia. Z Gastroenterol 2002;40:401-408.

\section{Beeinflussbarkeit funktioneller Darmstörungen über die Körperdecke - naturheilkundliche Konzepte}

Prof. Dr. med. Christine Uhlemann Klinik Innere Medizin II, Kompetenzzentrum Naturheilverfahren 07740 Jena

Allgemeine Wirkprinzipien der Naturheilverfahren (NHV) bzw. der naturheil kundlichen Medizin implizieren diese als Regulationsmedizin zur Beeinflussung von Autoregulationsmechanismen, zur Normalisierung der Körperfunktionen, d.h. physiologischer Regelsysteme, zur Kompensierung von Fehlfunk tionen und zur Initiierung von «Heilungsprozessen». NHV berücksichtigen er weiternd $\mathrm{zu}$ den sog. schulmedizinischen Konzepten konstitutionelle und nosologische (reflektorische) Bezüge.

Nosologische Bezüge vereinen reflektorische Beziehungen (a) zwischen den einzelnen Organsystemen (viszero-viszeral); (b) zwischen inneren Organsystemen und Strukturen der Körperoberfläche (viszero-kutan, -subkutan, -muskulär, -periostal), das entspricht den Head'schen, den Dicke'schen, den McKenzie'schen und den Periostzonen; (c) eine peripher-viszeral-zentrale Achse, die sich in der sog. Vagusafferenz darstellt im Sinne somatopsychischer Affektionen, d.h. periphere somatische Irritationen können zu psychischen Veränderungen, Störungen der Gestimmtheit mit negativen Emotionen führen. De Begriff der Vagusafferenz, die für zahlreiche unspezifische Schmerzzustände eine Erklärung sein könnte, ist dabei ein relevanter Fakt. Die Tatsache dieser neurophysiologischen Verschaltungen mit efferenten parasympathischen und sympathischen Einflüssen, einerseits als horizontales Prinzip, d.h. InnenAussen-, Aussen-Innen-Beziehung (Dermatom, Myotom, Sklerotom, Viszerotom) und andererseits als vertikales Prinzip, d.h. Oben-Unten-Beziehung (Sympathikus - Vagus - Stammhirn - Kortex, Meridiane, Fussreflexzonen sog. Fitzgeraldlinien) berechtigt, sog. ganzheitliche Reflexzonentherapien als Regulationstherapie gestörter viszeraler Funktionen anzuwenden.

Die Ebenen der Einflussnahme sind (a) Reflexzonen, z.B. Bindegewebszonen, Gelosen, Dermatome, Myotome, Sklerotome; (b) Somatotopien am Schädel, Ohr, an der Hand, am Fuss, Mund; (c) Meridiane, die in der Akupunktur, Akupressur, Moxibustion und Tuina (chinesische Massage im Meridianverlauf) genutzt wurden

Folgende naturheilkundliche Optionen sind bei einer gestörten Darmfunktion zweckmässig: unter Ausnutzung kutaneo-viszeraler Reflexe sind warme bis heisse Auflagen sowie sog. heisse Kurzwickel (Areal: Axilla bis Hüfte) nach Kneipp und Lumbalgüsse (Irritation der Head'schen Zone) für eine Durchblutungsförderung, damit Trophikbeeinflussung (Funktionsregulation) und viszerale Spannungsabnahme zweckmässig. Eine andere Option, die die Haut als Zielstruktur für reflektorisch regulative Strategien nutzt, sind die Akupunktur und die Fussreflexzonentherapie.

Dass in der Akupunkturphilosophie der «Bauch als interdisziplinäres Zentrum des Menschen» gesehen wird, drückt sich auch darin aus, dass auf der Bauchdecke die Zonen für die 5 Elemente (5 Wandlungsphasen) repräsentiert sind. In klinischen Studien konnte im Rahmen einer Meridiantherapie und unter Nutzung energetischer Punkte eine signifikante Verbesserung der Reizdarm symptomatik nach Verumakupunktur im Vergleich mit Scheinakupunktur nachgewiesen werden. In eigenen Untersuchungen wurde Akupunkturtherapie verglichen mit einer Bindegewebsmassage bei Patienten mit Fibromyalgiesyndrom, bei denen das Reizdarmsyndrom (RDS) ein pathognomonisches Phänomen ist. Im Ergebnis hat die Akupunktur (10 Mal, $2 \times$ wöchentlich) im Vergleich zur Bindegewebsmassage das RDS nur im Trend verbessert, während die Bindegewebsmassageserie $(10 \mathrm{Mal}, 2 \times$ wöchentlich) ein signifikante Reduktion der Reizdarmsymptome ergab.

Bei der Fussreflexzonentherapie, werden Reflexzonen (Fitzgeraldzonen) am Fuss stimuliert, die dem Organ zugeordnet sind, um im Sinne einer Autoregulation die gestörte Funktion zu beeinflussen. Dabei steht bis heute der neurophysiologische Beleg für diese Fussreflexzonen aus. In der täglichen Praxis weist sich die Fussreflexzonentherapie besonders bei funktionellen Störungen des Magen-Darm-Traktes durch eine empirische Wirksamkeit aus. 
Eine weitere probate Massnahme zur Beeinflussung einer gestörten Darmfunktion ist die Bindegewebsmassage, bei der mit einer spezifischen Grifftechnik (Schneidegriffe, Unterhautfasziengriff) nach einer vorgegebenen Systematik veränderte Zonen im Bindegewebe korrespondierend zum viszeralen Organ (viszerokutaner Reflex) beeinflusst werden. Die Reizung von sensorischen Afferenzen im Unterhautbindegewebe, die vom sympathischen Nervengeflecht umgeben sind, bedingen auch eine Änderung der Reaktionslage des Sympathikus.

Über die im Bindegewebe gelegenen Gelosen (prallelastische oder scharfkantige Verhärtungen im Bindegewebe als Fülle- oder Leeregelosen), sog. Schröpfzonen nach Abele, kann über ausleitende, im humoralpathologischen Sinne antidyskratische Verfahren, z.B. Schröpfen (blutig bzw. unblutig), Baunscheidtieren, Blutegel, Cantharidenpflaster, der Bindegewebsstoffwechsel in der entsprechenden darmassoziierten Bindegewebszone am Rücken angeregt werden und die Gelosen (Schnittstellen von vertikalen und horizontalen neurophysiologischen Verschaltungen) gelöscht bzw. beseitigt werden.

Muskeltechniken zur Nutzung des viszero-muskulären Reflexbezuges, um eine gestörte Darmfunktion zu beeinflussen, sind klassische Massagegrifftechniken in den zum Darm assoziierten Segmenten zur Triggerpunktbehandlung bzw. krankengymnastische Techniken im Sinne der postisometrischen Relaxation zur Beeinflussung der Muskelfehlspannung, die eine Korrespondenz zum Darm hat (M. piriformis, M. erector trunci, M. quadratus lumborum). Letztlich sind unter Nutzung des viszero-periostalen Bezuges auch manuelle Mobilisationen zweckmässig, die mit dem Darm korrespondierende Gelenkfunktionsstörungen beeinflussen (Iliosakralgelenke, Wirbelgelenke L2 - S1).

Zur Beeinflussung einer gestörten viszeralen Motilität ist die Kolonmassage nach Vogler und Krauss eine spezifische, punktuelle Grifftechnik, unter Be achtung des Atemrhythmus (5-Punkt-Behandlung über Kolon aszendens, Kolon transversum und Kolon deszendens, Sigma, Rektum) das intramural Nervengeflecht zu stimulieren und somit die Autoregulation zu fördern. Der eindeutige Wirksamkeitsnachweis steht noch aus.

Spezielle Techniken der Osteopathie (viszerale Techniken) sollen die Mobilität zwischen den Darmschlingen beeinflussen und somit auch die Autoregulation, d.h. das intramurale Geflecht und damit die Autarkie des Darmnervensystems erleichtern.

Zusammenfassend sind folgende Interventionen als Körperdeckenbeeinflussung einer gestörten Darmfunktion mit Angabe der Zielstruktur und der korrespondierenden Methoden aufgeführt (Tab. 1).

Tab. 1. Interventionen an der Körperdecke zur Beeinflussung einer gestörten Darmfunktion

\begin{tabular}{|c|c|}
\hline Zielstruktur & Methode \\
\hline Haut & $\begin{array}{l}\text { Kneipp'sche Hydrotherapie (Auflage, Wickel, Güsse) } \\
\text { Akupunktur } \\
\text { Fussreflexzonentherapie }\end{array}$ \\
\hline Bindegewebe & $\begin{array}{l}\text { Bindegewebsmassage } \\
\text { Lymphdrainage } \\
\text { Ausleitende Verfahren (z.B. Schröpfen) }\end{array}$ \\
\hline Muskel & $\begin{array}{l}\text { Krankengymnastik (PIR-Technik) } \\
\text { Klassische Massage (Triggerpunktbehandlung) } \\
\text { Thermische Hygiene }\end{array}$ \\
\hline $\begin{array}{l}\text { Gelenk } \\
\text { Viszerum }\end{array}$ & $\begin{array}{l}\text { Manuelle Mobilisation/Manipulation } \\
\text { Colonmassage } \\
\text { Viszerale Technik der Osteopathie }\end{array}$ \\
\hline
\end{tabular}

\section{Literatur}

1 Bühring M: Naturheilverfahren; in Zenz M, Jurna I: Lehrbuch der Schmerztherapie. Stuttgart, WVG, 2001.

2 Matejka R: Ausleitende Therapieverfahren, ed 2. Jena, Urban und Fischer, 2003

3 Mur E: Klassische Naturheilkunde. Forsch Komplementärmed 2001;8:86-89.

4 Schifter R, Harms E: Bindegewebsmassage. Stuttgart, Georg Thieme, 2005.

\section{Inhalte und Effizienz einer Fastentherapie}

\section{Dr. med. Rainer Stange}

Berlin

Die Fastentherapie ist ein essentieller Bestandteil im Selbstverständnis der klassischen Naturheilverfahren in Mitteleuropa sowohl in ihrer theoretischen Konzipierung wie ihrer praktischen Umsetzung. Allein in Deutschland werden derzeit mindestens 10000 vollstationäre Therapien mit Heilfasten als einem wesentlichen Bestandteil durchgeführt. Eine wesentlich grössere, bislang aber wissenschaftlich nicht ermittelte Zahl von Bundesbürgern fastet auf eigene Initiative oder in begleiteten Gruppen überwiegend in präventiver Intention. Im Kontrast zu dieser sehr weiten Verbreitung sind wir in der wissenschaftlichen Bewertung des Fastens eher auf fragmentierte Beobachtungen pathophysiologischer Effekte [3] auch aus Tierversuchen, ferner unkontrollierte oder kaum kontrollierte Studien angewiesen. Hieraus lassen sich jedoch eine ganze Reihe interessanter Effekte beschreiben, die für eine klinische Wirksamkeit sprechen. Der Wirksamkeitsnachweis ist bislang nur einigermassen zufrieden stellend für die chronische Polyarthritis gelöst.

Wichtige Elemente einer ärztlich geführten Fastentherapie, die letztlich auch zu ihrem Erfolg wesentlich beitragen, sind: Beurteilung von Indikation, Kontraindikationen, unerwünschten Ereignissen; teilstandardisierter Ablauf: Darmentlastung, definierte Fastengetränke; begleitend Hydro-/Thermotherapie (Reizsteigerung); begleitend Bewegungstherapie (körperliche Aktivität und Massagen, insbesondere Bindegewebsmassagen); Ordnungstherapie (Gespräche, Fastengruppe, Psychotherapie). Struktur- und Prozessqualität für ärztlich geleitete ambulante und stationäre Fastentherapien sind von der einzigen Fachgesellschaft im deutschsprachigen Raum in einem Konsensus-Prozess erarbeitet und publiziert worden [1].

1. Indikationsgebiet funktionelle Magen-Darm-Erkrankungen

Die temporäre Entlastung des Magen-Darm-Traktes hat nach den Beobachtungen erfahrener Fastenärzte hier dramatisch günstig Folgen, sowohl bei der «Non-ulcer Dyspepsia» im oberen wie dem Reizdarmsyndrom (RDS) im unteren Verdauungstrakt. Eine sehr wirkungsvolle Unterstützung stellt die manuelle Bauchbehandlung nach F.X. Mayr dar. Für die Mayr-Ärzte ist dies sogar das wichtigste Indikationsgebiet, da sie reversible Veränderungen im mesenterialen Entsorgungssystem (das so genannte Radix-Ödem) als Korrelat postulieren. Die klinische Forschung steht hier noch ganz am Anfang, obwohl die Epidemiologie, das fehlende Angebot der konventionellen Medizin und der zunehmende Anspruch der Patienten auf wirksame Behandlung grosse Möglichkeiten eröffnen. Die Vorstellung alter Fastenärzte, nach denen der symptomatische Verdauungstrakt genauso geschont und schrittweise wiederbelastet werden kann und sollte, wie wir dies sonst in der Medizin mit jedem anderen dysfunktionalen oder gar traumatisierten Körperteil durchführen, hat in Tierversuchen ansatzweise eine Bestätigung erfahren [4]. Das Modell des Lachses, der über seine ganze Lebensspanne jährlich lange Fastenphasen (bis 110 Tage) einlegen muss, zeigt eine schnell einsetzende, drastische, jedoch vollreversible Reduktion gastrointestinaler, insbesondere auch prokinetischer Hormone.

\section{Indikationsgebiet chronisch-entzündliche Gelenkerkrankungen}

Spätestens seit den auch international anerkannten Verdiensten einer diätetischen Behandlung der chronischen Polyarthritis z.B. durch Max Bircher-Benner und durch den grossen Fastenarzt Otto Buchinger d. Ä. [2] in den 20erund 30er-Jahren des letzten Jahrhunderts hat sich diese Indikation fest etablieren lassen. Auch in Skandinavien setzte sich diese Therapie durch und wurde zunehmend erforscht. Wir überblicken heute mehrere kontrollierte Studien, die in einem Review zusammengefasst wurden [5, 6]. Offenbar sind ein initiales Fasten und eine anschliessende relativ strikt geführte Ernährung (unter anderem temporär vegetarisch und glutenfrei unter sorgsamer Beobachtung von Triggereffekten aus der Ernährung) in der Lage, über mindestens 1 Jahr klinische Effekte einschliesslich Reduktion der Entzündungsparameter zu erzielen die einem Vergleich mit einem «Disease Modyfying Anthirheumatic Drug» (DMARD, früher etwa «Basistherapie») standhalten [7]. Als immunsuppressiver Wirkmechanismus wird hier auch eine dauerhafte Änderung des gastrointestinalen Milieus diskutiert.

\section{Literatur}

1 Ärztegesellschaft Heilfasten und Ernährung e.V, ExpertInnengruppe der ÄGHE: Leitlinien zur Fastentherapie. Forsch Komplementärmed Klass Naturheilkd 2002;9:189-198.

2 Buchinger O: Das Heilfasten und seine Hilfsmethoden als biologischer Weg, ed 23. Stuttgart, Hippokrates, 1999.

3 Fahrner H: Fasten als Therapie, ed 2. Stuttgart, Hippokrates, 1985.

4 Krogdahl A, Bakke-McKellep AM: Fasting and refeeding cause rapid changes in intestinal tissue mass and digestive enzyme capacities of Atlantic salmon (Salmo salar L.). Comp Biochem Physiol A Mol Integr Physiol 2005;141:450-460.

5 Kjeldsen-Kragh J et al : Controlled trial of fasting and one-year vegetarian diet in rheumatoid arthritis. Lancet 1991;338:899-902.

6 Müller H , Wilhelmi de Toledo FW, Resch KL: Fasting followed by vegetarian diet in patients with rheumatoid arthritis: a systematic review. Scand J Rheum 2001;30:1-10.

7 Peltonen $\mathrm{R}$ et al: Changes of fecal flora during fasting and one-year of vegetarian diet. Br J Rheumatol 1994;33:638-643. 